\title{
Right-Angled Differential Transmission Line Using Un-Chamfered Inductive Layout
}

\author{
Chia-Han Chang, Wen-Ju Chen, and Chun-Long Wang \\ Electronics Engineering, National Taiwan University of Science and Technology \\ 106 Keelung Rd., Sec. 4, No. 43, Taipei, Taiwan \\ M9602247@mail.ntust.edu.tw; sabrina19960214@gmail.com; clw@mail.ntust.edu.tw
}

\begin{abstract}
In this paper, the right-angled differential transmission line, the right-angled differential transmission line using the chamfered inductive layout, and the right-angled differential transmission line using the un-chamfered inductive layout are presented and compared. The right-angled differential transmission line has a very large value of differential-to-common mode conversion, which is found to be $-7.27 \mathrm{~dB}$ from $0.1 \mathrm{GHz}$ to $5.5 \mathrm{GHz}$. By using the chamfered inductive layout, the differential-to-common mode conversion can be kept below $-14.75 \mathrm{~dB}$ from $0.1 \mathrm{GHz}$ to $5.5 \mathrm{GHz}$. By using the un-chamfered inductive layout, the differential-to-common mode conversion can be further kept below $-21.70 \mathrm{~dB}$ from $0.1 \mathrm{GHz}$ to $5.5 \mathrm{GHz}$.
\end{abstract}

Keywords: Right-angled, Differential transmission line, Differential-to-common mode conversion, Chamfered inductive layout, Un-chamfered inductive layout.

\section{Introduction}

Due to the increasing complexity of the digital system and the decreasing rise time of the digital signal, the interference between the signal traces on the printed circuit board (PCB) layout is becoming more and more serious, causing digital signal distortion and malfunctioning of the logic gates. In order to overcome this problem, some researchers suggest that the differential transmission line should be applied on the PCB layout in order to suppress the common-mode noise interference between the signal traces on the PCB layout [1]-[6]. First of all, the differential transmission line is used to alleviate the influence of the non-ideal return path, reducing the electromagnetic interference caused by the common-mode noise current. Besides, the differential transmission line is applied to reduce the ground bounce arising from the microstrip line passing over the slotline [3]. Furthermore, the differential transmission line is adopted to reduce the interference between neighbouring transmission lines on the PCB layout [4]-[6].

However, since integrated circuit (IC) components are randomly distributed on the PCB, the circuit routing of the differential transmission line should detour the IC components, forming the right-angled differential transmission line as shown in Fig. 1. The right-angled differential transmission line would give rise to the common-mode noise even though the differential transmission line is utilized to suppress the common-mode noise. In order to tackle the common-mode noise of the differential transmission line, some researchers have proposed various techniques [7]-[10]. First of all, a common-mode filter formed by the periodic dumbbell slots in the ground plane is proposed [7]. The slots in the ground plane would block the return current of the common-mode noise, causing the common-mode noise to be filtered out at a designated frequency band while the differential-mode signal would not be affected by the slots in the ground plane since its return current would not find its path in the ground plane. The advantage of this structure is that its cost would be low since the conventional PCB fabrication process could be used to realize this structure. However, a large area would be consumed by the periodic dumbbell slots in the ground plane. In order to enhance the bandwidth and reduce the circuit size, a common-mode filter formed by a conjunction of $U$-shaped and $H$-shaped slots in the ground plane is proposed [8]. Although the bandwidth is enhanced and the circuit size is reduced, ground bounce would be induced when the differential transmission line passes over the slots in the ground plane, causing power integrity problems. In order to further reduce the circuit size, a right-angled differential transmission line using the compensation capacitance is proposed [9]. Although the circuit size would be greatly reduced, the cost would be increased when the compensation capacitance is implemented with a surface mounted device (SMD) capacitor. In duality, a right-angled differential transmission line using the compensation inductance is proposed as shown in Fig. 2 [10]. Both the common-mode noise could be efficiently reduced and the cost could be kept low since a conventional 
PCB fabrication process could be utilized. In order to further reduce the common-mode noise, a right-angled differential transmission line using the un-chamfered inductive layout is proposed as shown in Fig. 3.

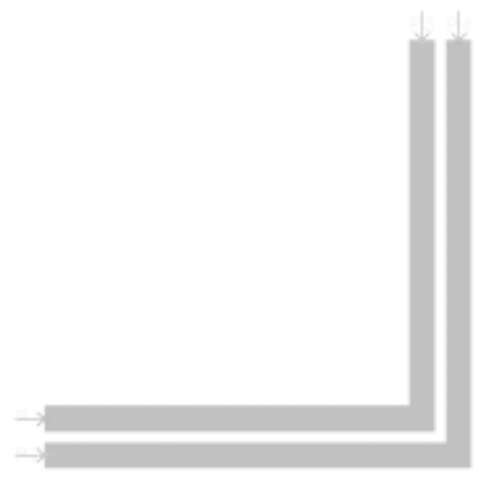

Fig. 1: The schematic view of the right-angled differential transmission line.

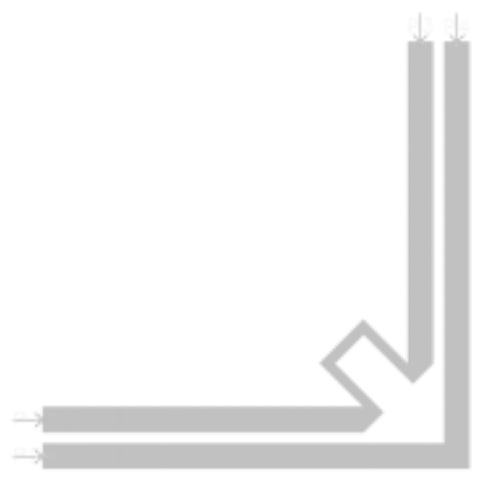

Fig. 2: The schematic view of the right-angled differential transmission line using the chamfered inductive layout.

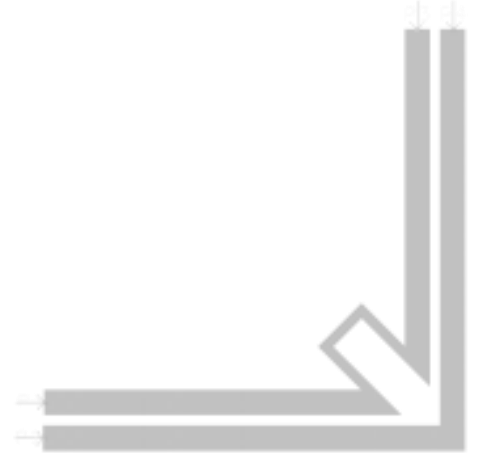

Fig. 3: The schematic view of the right-angled differential transmission line using the un-chamfered inductive layout.

\section{Differential-to-Common Mode Conversions for Various Types of Right-Angled Differential Transmission Lines}

In order to investigate the differential-to-common mode conversion, the right-angled differential transmission line shown in Fig. 1, the right-angled differential transmission line using the chamfered inductive layout shown in Fig. 2, and the right-angled differential transmission line using the un-chamfered inductive layout shown in Fig. 3 are simulated by (Advanced Design System) ADS. The dimensions for the right-angled differential transmission line shown in Fig. 1 
and that shown in Fig. 2 could be found in the literature [10]. The corresponding frequency responses of the differential-tocommon mode conversions for these structures are shown in Fig. 4. As can be seen from Fig. 4, the value of the differentialto-common mode conversion for the right-angled differential transmission line is smaller than $-7.27 \mathrm{~dB}$ from $0.1 \mathrm{GHz}$ to 5.5 GHz. The value of the differential-to-common mode conversion is very large since there is electrical length difference between the inner path and outer path of the differential transmission line as can be seen from Fig. 1. In order to compensate for the electrical length difference between the inner path and outer path of the differential transmission line, a right-angled differential transmission line using the chamfered inductive layout is proposed as shown in Fig. 2. As can be seen from Fig. 4, the right-angled differential transmission line using the chamfered inductive layout can keep the differential-to-common mode conversion below $-14.75 \mathrm{~dB}$ from $0.1 \mathrm{GHz}$ to $5.5 \mathrm{GHz}$. The low value of the differential-to-common mode conversion is evident from Fig. 2 since the inner and outer paths of the differential transmission line are now made similar in electrical length. In order to further reduce the differential-to-common mode conversion, a right-angled differential transmission line using the un-chamfered inductive layout is proposed as shown in Fig. 3. As can be seen from Fig. 4, the right-angled differential transmission line using the un-chamfered inductive layout can further keep the differential-to-common mode conversion below $-21.70 \mathrm{~dB}$ from $0.1 \mathrm{GHz}$ to $5.5 \mathrm{GHz}$. As can be observed from Fig. 3, since the un-chamfered part would provide excess capacitance to compensate for the electrical length difference between the inner and outer paths of the differential transmission line, the inner and outer paths of the differential transmission line would now be made much more similar in electrical length, causing the differential-to-common mode conversion to further drop down.

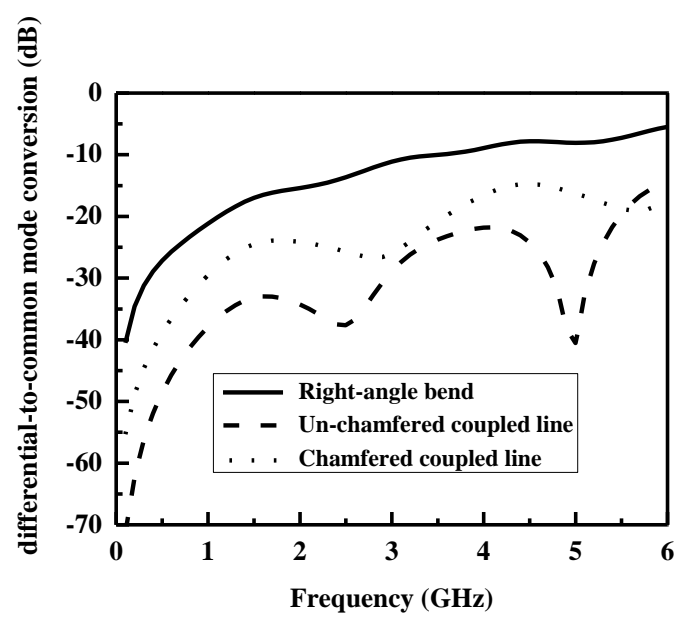

Fig. 4: Comparison between the simulated differential-to-common mode conversions for the right-angled differential transmission line, the right-angled differential transmission line using the chamfered inductive layout, and the right-angled differential transmission line using the un-chamfered inductive layout.

\section{Verifications}

In order to verify the simulation results, the right-angled differential transmission line using the chamfered inductive layout shown in Fig. 2 is implemented as shown in Fig. 5 while the right-angled differential transmission line using the unchamfered inductive layout shown in Fig. 3 is implemented as shown in Fig. 6. Fig. 5 and Fig. 6 are then measured by the Agilent/E5071B after it is calibrated with the N4431A electronic calibration kit. Fig. 7 shows the comparison between the simulated and measured differential-to-common mode conversions for the right-angled differential transmission line using the chamfered inductive layout and the right-angled differential transmission line using the un-chamfered inductive layout. As can be seen from Fig. 7, the simulation and measurement results are in good agreement for both the right-angled differential transmission line using the chamfered inductive layout or that using the un-chamfered inductive layout. 


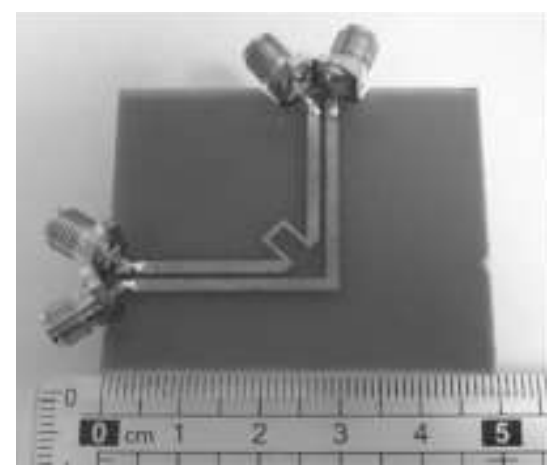

Fig. 5: Photograph of the right-angled differential transmission line using the chamfered inductive layout.

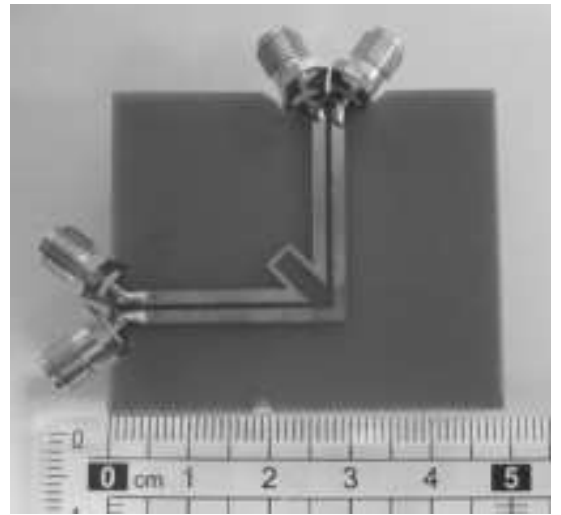

Fig. 6: Photograph of the right-angled differential transmission line using the un-chamfered inductive layout.

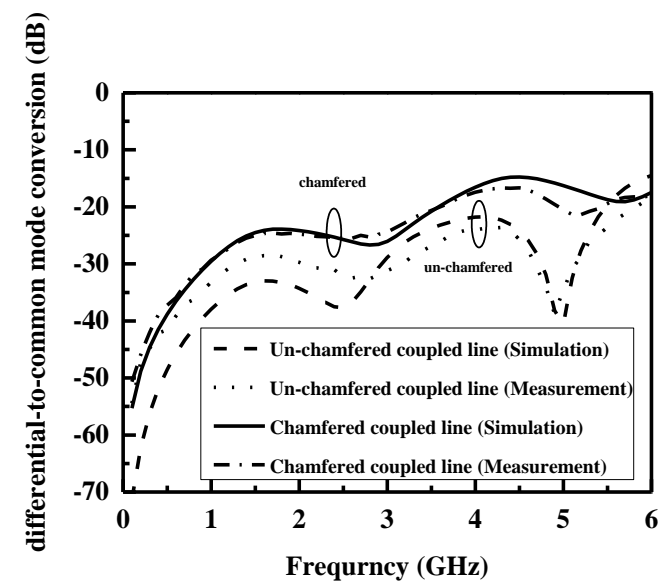

Fig. 7: Comparison between the simulated and measured differential-to-common mode conversions for the right-angled differential transmission line using the chamfered inductive layout and the right-angled differential transmission line using the un-chamfered inductive layout.

\section{Conclusion}

The differential-to-common mode conversion for the right-angled differential transmission line is investigated and found to be $-7.27 \mathrm{~dB}$, which is very large. By applying the right-angled differential transmission line using the chamfered inductive layout, the differential-to-common mode conversion would be greatly reduced to $-14.75 \mathrm{~dB}$. By applying the right-angled differential transmission line using the un-chamfered inductive layout, the differential-to-common mode conversion would be further reduced to $-21.70 \mathrm{~dB}$. 


\section{Acknowledgements}

This work was supported in part by the Ministry of Science and Technology, Taiwan, under Grant MOST-107-2221-E011-052.

\section{References}

[1] S. H. Hall, G. W. Hall, and J. A. McCall,High-Speed Digital System Design. New York: Wiley, 2000, pp. 140-141.

[2] P. E. Fornberg, M. Kanda, P. M. Melinda, and H. H. Stephen, "The impact of a nonideal return path on differential signal integrity," IEEE Trans. Electromagn. Compat., vol. 44, pp. 671-676, Feb.2002.

[3] G. H. Shiue and R. B. Wu, "Reduction in reflections and ground bounce for signal line through a split power by using differential coupled microstrip lines," IEEE 12th Topical Meeting on Electrical Performance of Electronic Packaging, pp. 107-110, Princeton, New Jersey, USA, October 2003.

[4] Y. Massoud, J. Kawa, D. MacMillen, and J. White, "Modeling and analysis of differential signaling for minimizing inductive crosstalk," Proc. Design Automation Conf., 2001, pp. 804-809.

[5] E. P. Li, H. F. Jin, W. L. Yuan, and L. W. Li, "Parallelized computational technique for signal propagation analysis at very high speed differential transmission lines," in Proc. IEEE Int. Symp. Electromagn. Compat., 2003, pp. 850-854.

[6] W.D. Guo, G. H. Shiue, C.M. Lin, and R.B.Wu, "Comparisons between serpentine and flat spiral delay lines on transient reflection/transmission waveforms and eye diagrams," IEEE Transactions on Microwave Theory and Techniques, vol. 54, pp. 1379-1387, April 2006.

[7] W. T. Liu, C. H. Tsai, T. W. Han, and T. L. Wu, "An embedded common-mode suppression filter for GHz differential signals using periodic defected ground plane," IEEE Microwave and Wireless Components Letters, vol. 18, no. 4, pp. 248 -pp. 250, Apr. 2008.

[8] S. J. Wu, C. H, Tsai, T. L. Wu, and T. Itoh, "A novel wideband common-mode suppression filter for gigahertz differential signals using coupled patterned ground structure," IEEE Transactions on Microwave Theory and Techniques, vol. 57, no.4, pp. 848-855, Apr. 2009.

[9] G. H. Shiue, W. D. Guo, C. M. Lin, and R.-B. Wu, "Noise reduction using compensation capacitance for bend discontinuities of differential transmission lines," IEEE Transactions on Advanced Packaging, vol. 29, pp. 560-569, August 2006.

[10] C. H. Chang, R. Y. Fang, and C. L. Wang, "Bended differential transmission line using compensation inductance for common-mode noise suppression," IEEE Trans. Components, Packaging, and Manufacturing Technology, vol. 2, no. 9, pp. 1518-1525, Sep. 2012. 